In Electrostatics of Soft and Disordered Matter, D. S. Dean, J. Dobnikar, A. Naji, and R. Podgornik, Eds., Proceedings of the CECAM Workshop "New Challenges in Electrostatics of Soft and Disordered Matter" (Pan Stanford, 2013)

\title{
Coarse-Grained Modeling of Charged Colloidal Suspensions: From Poisson-Boltzmann Theory to Effective Interactions
}

\author{
Alan R. Denton \\ Department of Physics, North Dakota State University, Fargo, North Dakota, U.S.A. \\ alan.denton@ndsu.edu
}

\section{Introduction}

Electrostatic forces - the strongest interparticle forces outside of the nucleus - account for the stability of matter over a broad range of length scales, from atoms to macromolecules to the myriad materials that surround us. Colloidal particles (nanometers to microns in size) and polymers can become charged in a polar solvent (e.g., water) through dissociation of counterions [1]. Repulsive Coulomb interactions between ions can then stabilize a suspension or solution against aggregation due to ubiquitous van der Waals attractive interactions [2]. Electrostatic interactions between ions largely govern the equilibrium thermodynamic and dynamical properties of charge-stabilized colloidal suspensions and polyelectrolyte solutions. Controlling mechanical and thermal stability is essential to many applications - from foods and pharmaceuticals to filters and photonic materials.

Predicting the properties of such complex, multicomponent systems with accuracy sufficient to guide and interpret experiments requires realistic modeling of the interparticle interactions and collective behavior of many-particle systems. While the fundamental interactions are simple, the sheer number of particles and the broad ranges of length and time scales confront the modeler with significant computational challenges. A general strategy for mitigating such challenges is to "coarse grain" or "integrate out" the degrees of freedom of some components, reducing the original multicomponent model to a simpler model of fewer components [3, 4, 5, 6]. The trade-off for so reducing complexity is that the simpler model is governed by modified (effective) interparticle interactions.

This chapter is a "how-to" manual of sorts for implementing coarse-graining methods to derive effective interactions. For simplicity, we focus here on charge-stabilized colloidal suspensions. The same basic concepts apply, however, to a wide variety of soft (and hard) materials. To set the stage, we begin by defining the primitive and one-component 
models and outlining the Poisson-Boltzmann (PB) theory of charged colloids. After reviewing the well-known cell model implementation of PB theory, we turn to an alternative implementation, based on perturbation theory, and derive microion distributions around colloids and effective electrostatic interactions. After some effort, a happy result emerges: the effective interactions remain relatively simple, at least in systems with monovalent microions. This fortunate outcome provides the foundation for further theoretical and simulation modeling to explore and facilitate design of novel materials. Finally, we peer over the horizon at the outlook for possible future research directions in the field.

\section{Primitive Model}

The primitive model of charged colloids and polyelectrolytes [1, 2] idealizes the solvent as a homogeneous dielectric continuum of relative permittivity $\epsilon$. Dispersed throughout the solvent are macroions and microions, modeled here as charged hard spheres of radius $a$ and valence $Z$ (charge $-Z e$ ) and point ions of valence $z$. In a closed suspension, all particles are confined to the same volume $V$. In Donnan equilibrium, only the macroions are confined, while the microions can exchange (e.g., across a semi-permeable membrane) with a salt reservoir, here assumed to be a 1:1 electrolyte of monovalent ions.
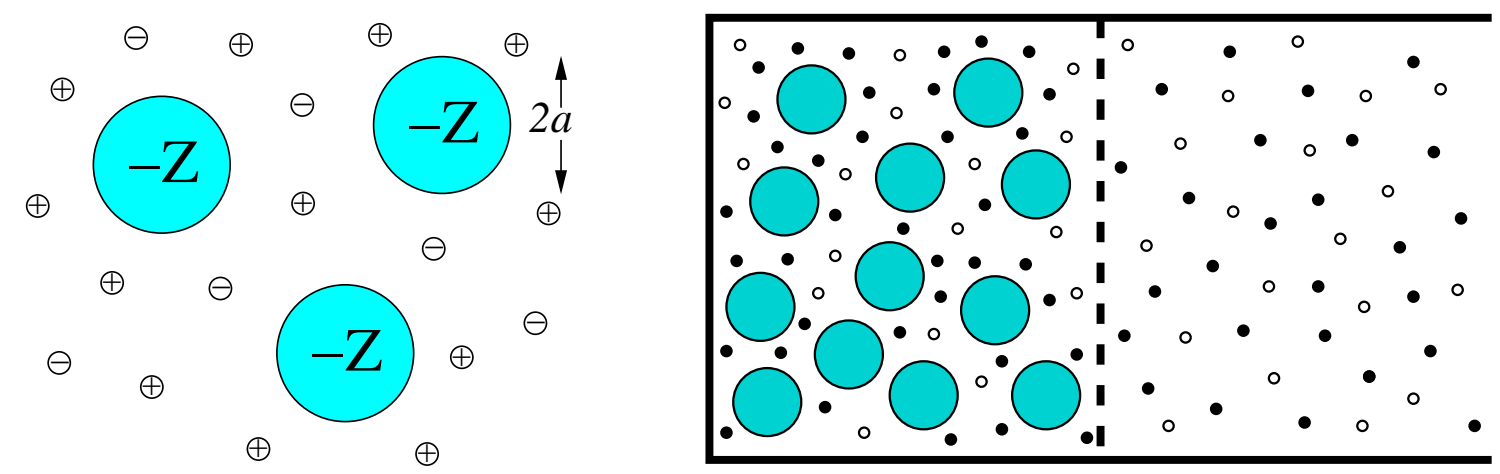

Figure 1. Primitive model of charged colloids: spherical macroions (radius $a$, valence $Z$ ) and point microions dispersed in a dielectric continuum (left). Colloidal suspension in Donnan equilibrium across semi-permeable membrane with electrolyte reservoir (right).

If sufficiently dilute, the reservoir can be reasonably modeled as an ideal gas. The reservoir fixes the chemical potential of salt in the suspension, $\mu_{s}=2 k_{B} T \ln \left(n_{0} \Lambda^{3}\right)$, where $n_{0}$ is the number density of ion pairs, $T$ the absolute temperature, and $\Lambda$ the thermal wavelength, defining the arbitrary zero of the chemical potential. A bulk suspension of $N_{m}$ macroions, $N_{c}$ counterions, and $N_{s}$ dissociated pairs of oppositely charged salt ions contains $N_{+}=N_{c}+N_{s}$ positive and $N_{-}=N_{s}$ negative microions, for a total of $N_{\mu}=N_{c}+2 N_{s}$ microions. Global electroneutrality constrains the macroion and counterion numbers by the condition $Z N_{m}=z N_{c}$. 
Accounting for excluded-volume and electrostatic (Coulomb) pairwise interparticle interactions, the Hamiltonian separates naturally into three terms:

$$
H=H_{m}+H_{\mu}+H_{m \mu} .
$$

The first term on the right side is the macroion Hamiltonian

$$
H_{m}=H_{\mathrm{hs}}+\frac{1}{2} \sum_{i \neq j=1}^{N_{m}} v_{m m}\left(r_{i j}\right),
$$

where $H_{\mathrm{hs}}$ is the hard-sphere Hamiltonian (including kinetic energy), $v_{m m}\left(r_{i j}\right)=Z^{2} \lambda_{B} / r_{i j}$ is the Coulomb pair potential between a pair of macroions with center-center separation $r_{i j}$, and $\lambda_{B}=e^{2} /\left(\epsilon k_{B} T\right)$ defines the Bjerrum length, the distance at which the Coulomb interaction energy between a pair of monovalent ions rivals the thermal energy. To simplify notation, in this chapter, Hamiltonians, pairpotentials, and all other quantities having dimensions of energy are expressed in thermal $\left(k_{B} T\right)$ units. The remaining two terms on the right side of Eq. (11) are the microion Hamiltonian

$$
H_{\mu}=K_{\mu}+\frac{\lambda_{B}}{2} \sum_{i \neq j=1}^{N_{\mu}} \frac{z_{i} z_{j}}{r_{i j}},
$$

with kinetic energy $K_{\mu}$ and microion valences $z_{i}= \pm 1$, and the macroion-microion interaction energy

$$
H_{m \mu}=Z \lambda_{B} \sum_{i=1}^{N_{m}} \sum_{j=1}^{N_{\mu}} \frac{z_{j}}{r_{i j}}
$$

\section{One-Component Model: Effective Hamiltonian}

Significant concentrations of salt pose severe computational challenges for large-scale simulations of bulk suspensions [7, 8, 9]. Consider that a suspension of macroions of radius $a=10 \mathrm{~nm}$ at $1 \%$ volume fraction and just $1 \mathrm{mM}$ salt concentration contains, per macroion, $\mathcal{O}\left(10^{3}\right)$ particles, all interacting via long-range Coulomb forces. Therefore, salt-dominated suspensions usually are modeled by first mapping the mixture onto a one-component model (OCM).

For a suspension in Donnan equilibrium, this mapping (or coarse graining) operates on the semigrand partition function:

$$
\mathcal{Z}=\left\langle\langle\exp (-H)\rangle_{\mu}\right\rangle_{m}
$$

where \langle\rangle$_{\mu}$ denotes a grand canonical trace over microion coordinates and \langle\rangle$_{m}$ a canonical trace over macroion coordinates. The partition function may be formally expressed in the form

$$
\mathcal{Z}=\left\langle\exp \left(-H_{\text {eff }}\right)\right\rangle_{m}
$$


where

$$
H_{\text {eff }}=H_{m}+\Omega_{\mu}
$$

is an effective Hamiltonian of a one-component system of pseudo-macroions and

$$
\Omega_{\mu}=-\ln \left\langle\exp \left(-H_{\mu}-H_{m \mu}\right)\right\rangle_{\mu}
$$

is the grand potential functional of the microions in the presence of fixed macroions. Practical applications of the OCM require approximating $\Omega_{\mu}$. For this purpose, Poisson-Boltzmann

(PB) theory [1, 2, 10, 11] is a powerful approach. Below we briefly review PB theory and two common implementations: the cell model and the effective-interaction model.

\section{Poisson-Boltzmann Theory}

Poisson-Boltzmann theory is most elegantly formulated within the framework of classical density-functional theory of nonuniform fluids [12, 13, 14, 15, 16]. Corresponding to the primitive model Hamiltonian [Eqs. (2)-(4)], there exists a Helmholtz free energy functional $F\left[n_{m}(\mathbf{r}), n_{ \pm}(\mathbf{r})\right]$, which (for a given external potential) is a unique functional of the macroion and microion number density profiles, $n_{m}(\mathbf{r})$ and $n_{ \pm}(\mathbf{r})$, varying with position $\mathbf{r}$ [13]. This free energy functional separates, according to $F=F_{\text {id }}+F_{\text {ex }}+F_{\text {ext }}$, into a (purely entropic) ideal-gas free energy functional of all ions,

$$
F_{\text {id }}=\int \mathrm{d} \mathbf{r} \sum_{i=m, \pm} n_{i}(\mathbf{r})\left\{\ln \left[n_{i}(\mathbf{r}) \Lambda^{3}\right]-1\right\},
$$

an excess free energy functional, $F_{\text {ex }}=F_{\mathrm{hs}}+F_{\mathrm{el}}$, due to hard-sphere (hs) and electrostatic (el) interparticle interactions, and a contribution $F_{\text {ext }}$ due to an external potential. Neglecting interparticle correlations (mean-field approximation), the electrostatic part of the excess free energy functional may be approximated as

$$
F_{\text {el }}=\frac{1}{e} \int \mathrm{d} \mathbf{r} \rho(\mathbf{r}) \Psi(\mathbf{r}),
$$

where $\rho(\mathbf{r})=e\left[n_{+}(\mathbf{r})-n_{-}(\mathbf{r})-n_{f}(\mathbf{r})\right]$ is the total charge density, including the negative charge fixed on the macroion surfaces of number density $n_{f}(\mathbf{r})$, and

$$
\Psi(\mathbf{r})=\frac{\lambda_{B}}{e} \int \mathrm{d} \mathbf{r}^{\prime} \frac{\rho\left(\mathbf{r}^{\prime}\right)}{\left|\mathbf{r}-\mathbf{r}^{\prime}\right|}
$$

is the reduced electrostatic potential at position $\mathbf{r}$ due to all ions. The reduced potential and total ion density are related via the Poisson equation, which may be expressed in the form

$$
\nabla^{2} \psi(\mathbf{r})=-4 \pi \lambda_{B}\left[n_{+}(\mathbf{r})-n_{-}(\mathbf{r})\right] ;\left.\quad \nabla \psi\right|_{\text {surface }}=Z \lambda_{B} / a^{2},
$$

where the macroion charges are absorbed into a boundary condition at the macroion surfaces and the microion densities implicitly vanish inside the macroion cores.

In a given external potential, the equilibrium densities of all ions minimize the total grand potential functional of the system [13]. Alternatively, fixing the macroions and 
regarding their charges as the source of the external potential, the equilibrium microion densities alone minimize the microion grand potential functional

$$
\Omega_{\mu}\left[n_{ \pm}(\mathbf{r})\right]=F_{\mu}\left[n_{ \pm}(\mathbf{r})\right]-\mu_{+} \int \mathrm{d} \mathbf{r} n_{+}(\mathbf{r})-\mu_{-} \int \mathrm{d} \mathbf{r} n_{-}(\mathbf{r}),
$$

a Legendre transform of the microion free energy functional

$$
F_{\mu}\left[n_{ \pm}(\mathbf{r})\right]=F_{\mu, \text { id }}\left[n_{ \pm}(\mathbf{r})\right]+\frac{1}{2 e} \int \mathrm{d} \mathbf{r} \rho(\mathbf{r}) \Psi(\mathbf{r})
$$

where

$$
F_{\mu, \mathrm{id}}\left[n_{ \pm}(\mathbf{r})\right]=\int \mathrm{d} \mathbf{r} \sum_{i= \pm} n_{i}(\mathbf{r})\left\{\ln \left[n_{i}(\mathbf{r}) \Lambda^{3}\right]-1\right\}
$$

is the ideal-gas free energy functional and the microion (electro)chemical potentials $\mu_{ \pm}$ are identified as the Legendre variables. Note that $\Omega_{\mu}$ depends parametrically on the macroion coordinates and that $F_{\mu}$ includes macroion-macroion Coulomb interactions for electroneutrality. Under the assumption that either the electrostatic potential or the electric field vanishes everywhere on the boundary of the volume $V$, the microion free energy functional also may be expressed in the form

$$
F_{\mu}\left[n_{ \pm}(\mathbf{r})\right]=\int \mathrm{d} \mathbf{r} \sum_{i= \pm} n_{i}(\mathbf{r})\left\{\ln \left[n_{i}(\mathbf{r}) \Lambda^{3}\right]-1\right\}+\frac{1}{8 \pi \lambda_{B}} \int \mathrm{d} \mathbf{r}|\nabla \psi|^{2}
$$

Minimizing $\Omega_{\mu}\left[n_{ \pm}(\mathbf{r})\right]$ with respect to $n_{ \pm}(\mathbf{r})$ now yields the Boltzmann approximation for the equilibrium microion densities

$$
n_{ \pm}(\mathbf{r})=n_{ \pm}^{(0)} \exp [\mp \psi(\mathbf{r})] \quad \text { (fixed macroions) }
$$

where the reference densities, $n_{ \pm}^{(0)}=\Lambda^{-3} \exp \left(\mu_{ \pm}\right)$, are the microion densities at the reference potential $\psi=0$. The microion grand potential is the value of the grand potential functional [Eq. (13)] evaluated at the equilibrium density profiles [Eq. (17)]:

$$
\Omega_{\mu}=-\int \mathrm{d} \mathbf{r}\left[n_{+}(\mathbf{r})+n_{-}(\mathbf{r})\right]-\frac{1}{2} \int \mathrm{d} \mathbf{r}\left[n_{+}(\mathbf{r})-n_{-}(\mathbf{r})+n_{f}(\mathbf{r})\right] \psi(\mathbf{r}) .
$$

For a closed suspension (fixed particle numbers), the chemical potentials of the two microion species differ because of asymmetric interactions with the macroions: $\mu_{+} \neq \mu_{-}$. Correspondingly, the reference densities also differ: $n_{+}^{(0)} \neq n_{-}^{(0)}$. In Donnan equilibrium, however, exchange of microions with a salt reservoir shifts the intrinsic microion chemical potentials, $\mu_{ \pm}^{\text {in }}=\left[\delta F_{\mu} / \delta n_{ \pm}(\mathbf{r})\right]_{\mathrm{eq}}$, by the Donnan potential $\psi_{D}$ :

$$
\mu_{ \pm}=\mu_{ \pm}^{\text {in }} \pm \psi_{D}=\ln \left(n_{0} \Lambda^{3}\right) \text {. }
$$

The total chemical potentials, and so too the reference densities, of the two microion species are thus equalized. The equilibrium microion density profiles are then given by

$$
n_{ \pm}(\mathbf{r})=n_{0} \exp [\mp \psi(\mathbf{r})]
$$


The Donnan potential is interpreted physically as the change in electrostatic potential across the reservoir-suspension interface, and mathematically as a Lagrange multiplier for the constraint of global electroneutrality.

Combining the Poisson equation for the potential [Eq. (12)] with the Boltzmann approximation for the microion densities [Eq. (17) or (20)], the Poisson-Boltzmann equation becomes

$$
\nabla^{2} \psi(\mathbf{r})=\kappa_{0}^{2} \sinh \psi(\mathbf{r}) ;\left.\quad \nabla \psi\right|_{\text {surface }}=Z \lambda_{B} / a^{2},
$$

where $\kappa_{0}=\sqrt{8 \pi \lambda_{B} n_{0}}$ is the screening constant in the reservoir. Note that Eq. (21) is highly nonlinear. In the case of weak electrostatic potentials $(\psi \ll 1)$, the right side of Eq. (21) may be expanded in powers of $\psi$. Truncating at linear order yields the linearized PB equation:

$$
\nabla^{2} \psi(\mathbf{r})=\kappa_{0}^{2} \psi(\mathbf{r}) ;\left.\quad \nabla \psi\right|_{\text {surface }}=Z \lambda_{B} / a^{2} .
$$

Beyond the boundary condition at the macroion surfaces, the boundary-value problem is fully specified only by imposing another condition at the outer boundary of the system, which depends on the practical implementation of the theory.

\section{Cell-Model Implementation}

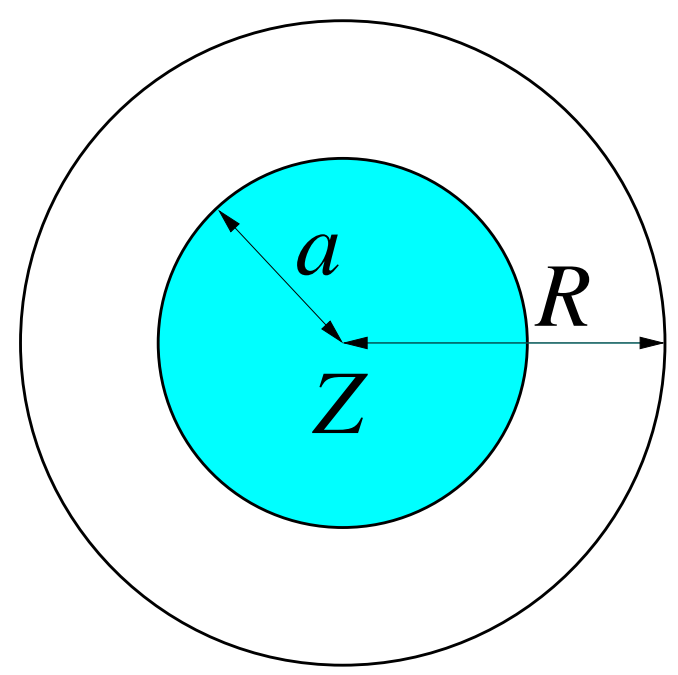

Figure 2. Cell model: a single macroion in a spherical cell.

The anisotropic boundary conditions on the nonlinear PB equation [Eq. (21)] imposed by an arbitrary configuration of macroions render a general solution computationally daunting. In recent years, powerful $a b$ initio methods have been developed for combining PB theory of microion density profiles with molecular dynamics [15, 16, 17, 18] or Brownian dynamics [19, 20] simulation to evolve macroion coordinates according to derived forces. 
Despite such advances, most applications of PB theory have been implemented within a cell model to facilitate numerical solution.

In a seemingly bold reduction, the cell model represents a bulk suspension by a single macroion, neutralizing counterions, and salt ions confined to a cell of the same shape as the macroion (see Fig. 2). Microion-induced correlations between macroions are simply ignored. For spherical colloids, the natural choice is a spherical cell centered on the macroion. Gauss's law then dictates that the electric field must vanish everywhere on the boundary of the electroneutral cell. With the potential and microion densities depending on only the radial coordinate $r$, the $\mathrm{PB}$ equation reduces to an ordinary differential equation for $\psi(r)$ with boundary conditions $\psi^{\prime}(a)=Z \lambda_{B} / a^{2}$ and $\psi^{\prime}(R)=0$, where the cell radius $R$ is commensurate with the average macroion density: $n_{m}=N_{m} / V=3 /\left(4 \pi R^{3}\right)$. For a closed suspension, the arbitrary location of the reference point of the electrostatic potential (where $\psi=0$ ) is usually chosen as the cell boundary: $\psi(R)=0$. In Donnan equilibrium, the potential is conventionally chosen to vanish in the reservoir, in which case the boundary value of the electrostatic potential is identified as the Donnan potential: $\psi(R)=\psi_{D} \neq 0$.

An appealing feature of the cell model is the simple analytic relation between the bulk pressure $p$ (in thermal units) and the microion densities at the cell boundary:

$$
p=n_{+}(R)+n_{-}(R) .
$$

Although first derived within the mean-field PB framework [21], this pressure theorem proves to be exact within the cell model [22, i.e., valid also for correlated microions. In Donnan equilibrium with an ideal-gas reservoir, the osmotic pressure $\Pi$, i.e., the difference in pressure between suspension and reservoir, is then given by

$$
\Pi=n_{+}(R)+n_{-}(R)-2 n_{0} .
$$

Within PB theory, the osmotic pressure is strictly positive [23], following from Eqs. (20) and (24) and the inequality, $\cosh x>1$.

The cell model provides one means of implementing PB theory by approximating the microion grand potential [Eq. (7)] in the one-component mapping of the primitive model (Sec. 3). Reducing a suspension to a single macroion in a cell with isotropic boundary conditions facilitates solution of the nonlinear PB equation. The cost of incorporating nonlinear microion screening, however, is neglect of correlations between macroions.

\section{Effective-Interaction Implementation}

An alternative implementation of PB theory, based on the one-component mapping, focuses on effective interactions derived from perturbative expansion of the microion grand potential about a reference system, namely, a uniform plasma of microions unperturbed by the macroions. By incorporating macroion interactions, this approach can model both thermodynamic and structural properties of colloidal suspensions. For reviews of effective interactions in colloidal suspensions, see refs. [3, 4, 5, 6]. Several statistical mechanical 
frameworks have been developed. Density-functional theories [24, 25, 26, 27] expand the ideal-gas part of the microion free energy functional [Eq. (15)] in a Taylor series in powers of deviations of the microion density profiles from their mean values $n_{ \pm}$:

$$
F_{\mu, \text { id }}\left[n_{ \pm}(\mathbf{r})\right]=\sum_{i= \pm}\left(N_{i}\left[\ln \left(n_{i} \Lambda^{3}\right)-1\right]+\int \mathrm{d} \mathbf{r} \frac{\left[n_{i}(\mathbf{r})-n_{i}\right]^{2}}{2 n_{i}}+\cdots\right)
$$

Distributon function theories are based on extensions of the Debye-Hückel theory of electrolytes [28]. Response theories [29, 30, 31, 32] are based on a similar perturbative expansion of the microion grand potential functional [Eq. (8)]:

$$
\Omega_{\mu}=\Omega_{0}+\int_{0}^{1} d \lambda\left\langle H_{m \mu}\right\rangle_{\lambda}
$$

where $\Omega_{0}=-\ln \left\langle\exp \left(-H_{\mu}\right)\right\rangle_{\mu}$ is the grand potential of a reference suspension in the absence of an external colloidal potential and $\left\langle H_{m \mu}\right\rangle_{\lambda}$ denotes an ensemble average of the macroion-microion interaction energy in a system in which the macroions are "charged" to a fraction $\lambda$ of their full charge, which can be related to the macroion-microion pair potentials $v_{m \pm}(r)= \pm Z \lambda_{B} / r$ and the densities, $n_{m}(\mathbf{r})$ and $n_{ \pm}(\mathbf{r})$, of macroions and microions:

$$
\left\langle H_{m \pm}\right\rangle_{\lambda}=\int d \mathbf{r} \int d \mathbf{r}^{\prime} v_{m \pm}\left(\left|\mathbf{r}-\mathbf{r}^{\prime}\right|\right) n_{m}(\mathbf{r})\left\langle n_{ \pm}\left(\mathbf{r}^{\prime}\right)\right\rangle_{\lambda} .
$$

Expanding the microion densities about a reference microion plasma in powers of the macroion "external" potential,

$$
\phi_{ \pm}(\mathbf{r})=\int \mathrm{d} \mathbf{r}^{\prime} v_{m \pm}\left(\left|\mathbf{r}-\mathbf{r}^{\prime}\right|\right) n_{m}\left(\mathbf{r}^{\prime}\right)
$$

and truncating the series at linear order yields the linear-response approximation

$$
n_{ \pm}(\mathbf{r})=\sum_{i= \pm} \int \mathrm{d} \mathbf{r}^{\prime} \chi_{ \pm i}\left(\left|\mathbf{r}-\mathbf{r}^{\prime}\right|\right) \phi_{i}\left(\mathbf{r}^{\prime}\right)
$$

The linear-response functions

$$
\chi_{i j}\left(\left|\mathbf{r}-\mathbf{r}^{\prime}\right|\right)=\left(\frac{\delta n_{i}(\mathbf{r})}{\delta \phi_{j}\left(\mathbf{r}^{\prime}\right)}\right)_{Z=0}
$$

describe the response of the reference plasma to the macroions and are related to the plasma pair correlation functions $h_{i j}(r)$ via 33 .

$$
\chi_{i j}(r)=-n_{i}\left[\delta_{i j} \delta(r)+n_{j} h_{i j}(r)\right] .
$$

The neglected higher-order terms in Eq. (29) involve nonlinear response functions and manyparticle correlations.

Approximating the pair correlation functions of the uniform plasma by their asymptotic (long-range) limits yields the so-called random phase approximation

$$
h_{i j}(r)=-z_{i} z_{j} \lambda_{B} \frac{e^{-\kappa r}}{r},
$$


where $\kappa=\sqrt{4 \pi \lambda_{B} n_{\mu}}$ is the screening constant in the system, which differs from that in the reservoir $\kappa_{0}$ [cf. Eq. (21)]. For consistency, $n_{\mu}$ here represents the total density of microions in the free volume, i.e., the volume not excluded by the macroion hard cores.

Inserting the linearized microion densities [Eq. (29)] into Eq. (27) recasts the effective Hamiltonian (microion grand potential) in the form of a sum of effective interactions:

$$
H_{\mathrm{eff}}=E_{v}+H_{\mathrm{hs}}+\frac{1}{2} \sum_{i \neq j=1}^{N_{m}} v_{\mathrm{eff}}\left(r_{i j}\right),
$$

where the volume energy $E_{v}$ is the microion grand potential for a single macroion, $v_{\text {eff }}(r)$ is an effective electrostatic pair potential between macroions, and neglected higher-order terms involve sums over effective many-body interactions. The volume energy takes the general form

$$
E_{v}=\Omega_{0}+\frac{1}{2} N_{m} v_{\text {ind }}(0)+\frac{1}{2}\left(N_{+}-N_{-}\right) \Psi_{D},
$$

where the first term on the right side accounts for the microion entropy, the second term for the macroion-microion interaction energy, and the last term is the Donnan potential energy resulting from the electroneutrality constraint. The effective macroion-macroion pair potential can be expressed as

$$
v_{\text {eff }}(r)=v_{m m}(r)+v_{\text {ind }}(r),
$$

which comprises the bare Coulomb pair potential $v_{m m}(r)$ and a microion-induced pair potential

$$
v_{\text {ind }}(r)=\int \mathrm{d} \mathbf{r}^{\prime}\left[n_{+}\left(r^{\prime}\right)-n_{-}\left(r^{\prime}\right)\right] v_{m+}\left(\left|\mathbf{r}-\mathbf{r}^{\prime}\right|\right) .
$$

To evaluate $v_{\text {ind }}(r)$, it is helpful first to consider the isotropic microion density profiles around a single macroion, which are obtained from Eqs. (29)-(32):

$$
\begin{aligned}
n_{ \pm}(r) & =\sum_{i= \pm} \int \mathrm{d} \mathbf{r}^{\prime} \chi_{ \pm i}\left(\left|\mathbf{r}-\mathbf{r}^{\prime}\right|\right) v_{m+}\left(r^{\prime}\right) \\
& = \pm n_{ \pm}\left(n_{\mu} \lambda_{B} \int d \mathbf{r}^{\prime} \frac{e^{-\kappa r^{\prime}}}{r^{\prime}} v_{m+}\left(\left|\mathbf{r}-\mathbf{r}^{\prime}\right|\right)-v_{m+}(r)\right) .
\end{aligned}
$$

To ensure exclusion of microions from the macroion hard cores, the macroion-microion pair potentials can be extended inside the core:

$$
v_{m \pm}(r)= \pm Z \lambda_{B} f(r) ; \quad f(r)= \begin{cases}1 / r, & r>a \\ \alpha / a, & r<a,\end{cases}
$$

where $\alpha$ is a constant to be chosen such that $n_{ \pm}(r)=0$ for $r<a$. After evaluating the convolution integral

$$
\int_{0}^{\infty} d r^{\prime} r^{\prime} e^{-\kappa r^{\prime}} g\left(r, r^{\prime}\right)=\frac{2}{\kappa^{2}} \begin{cases}\frac{1}{r}-\frac{e^{\kappa a}}{1+\kappa a} \frac{e^{-\kappa r}}{r}, & r>a \\ \frac{1}{a+\kappa^{-1}}, & r<a,\end{cases}
$$


with

$$
g\left(r, r^{\prime}\right)=\int_{-1}^{1} d \mu f\left(\left|\mathbf{r}-\mathbf{r}^{\prime}\right|\right) ; \quad \mu \equiv \mathbf{r} \cdot \mathbf{r}^{\prime} /\left(r r^{\prime}\right),
$$

one finds [from Eqs. (37) and (38)] $\alpha=\kappa a /(1+\kappa a)$ and

$$
n_{ \pm}(r)=\mp n_{ \pm} Z \lambda_{B}\left(\frac{e^{\kappa a}}{1+\kappa a}\right) \frac{e^{-\kappa r}}{r}, \quad r>a .
$$

Substituting Eq. (41) into Eqs. (35) and (36), and evaluating the integral (for $r>2 a$ )

$$
\int_{a}^{\infty} d r^{\prime} r^{\prime} e^{-\kappa r^{\prime}} g\left(r, r^{\prime}\right)=\frac{2}{\kappa^{2} r}\left(\frac{1+\kappa a}{e^{\kappa a}}-\frac{e^{\kappa a}}{1+\kappa a} e^{-\kappa r}\right),
$$

yields a screened-Coulomb (Yukawa) effective pair potential:

$$
v_{\text {eff }}(r)=Z^{2} \lambda_{B}\left(\frac{e^{\kappa a}}{1+\kappa a}\right)^{2} \frac{e^{-\kappa r}}{r}, \quad r>2 a .
$$

Finally, from Eqs. (36) and (41), we have

$$
v_{\text {ind }}(0)=-\frac{Z^{2} \lambda_{B}}{a+\kappa^{-1}},
$$

which allows the volume energy to be written more explicitly:

$$
E_{v}=\Omega_{0}-\frac{1}{2} N_{m} \frac{Z^{2} \lambda_{B}}{a+\kappa^{-1}}-\frac{1}{2} \frac{\left(N_{+}-N_{-}\right)^{2}}{N_{\mu}} .
$$

In passing, we recall that Eq. (43) is the basis of the classic Derjaguin-Landau-VerweyOverbeek (DLVO) theory [34, 35] of charged colloids, where it was first derived within a Debye-Hückel approximation, without the associated volume energy and without excludedvolume corrections.

It is important to remember that the one-body volume energy, although independent of macroion coordinates, does depend on the average macroion density, and therefore can influence thermodynamic properties. Similarly, the effective pair potential is densitydependent. Implications of density-dependent effective interactions for thermodynamic stability and consistency have been discussed extensively in recent years [36, 37, 38, 39, [40, 41].

Bulk thermodynamic and structural properties can be calculated ultimately by inputting the effective interactions, $E_{v}$ and $v_{\text {eff }}(r)$, into statistical mechanical theories or simulations of the OCM. Figures 3 and 4 show sample results for the osmotic pressure of highly charged colloidal suspensions, calculated using methodologies described in refs. [11, 42, 43]. Predictions of the PB cell and effective interaction (one-component) models agree closely with simulations of the primitive model [7] (Fig. 3) up to moderate electrostatic coupling strengths $\left(\Gamma \equiv \lambda_{B} / a<1\right)$ and with experimental data [44, 45] (Fig. 44) over a considerable range of colloid volume fractions. To achieve such agreement, the linear-response theory has been merged with charge renormalization theory to incorporate the important concept of an effective macroion valence [46]. By subsuming within the effective valence those counterions 


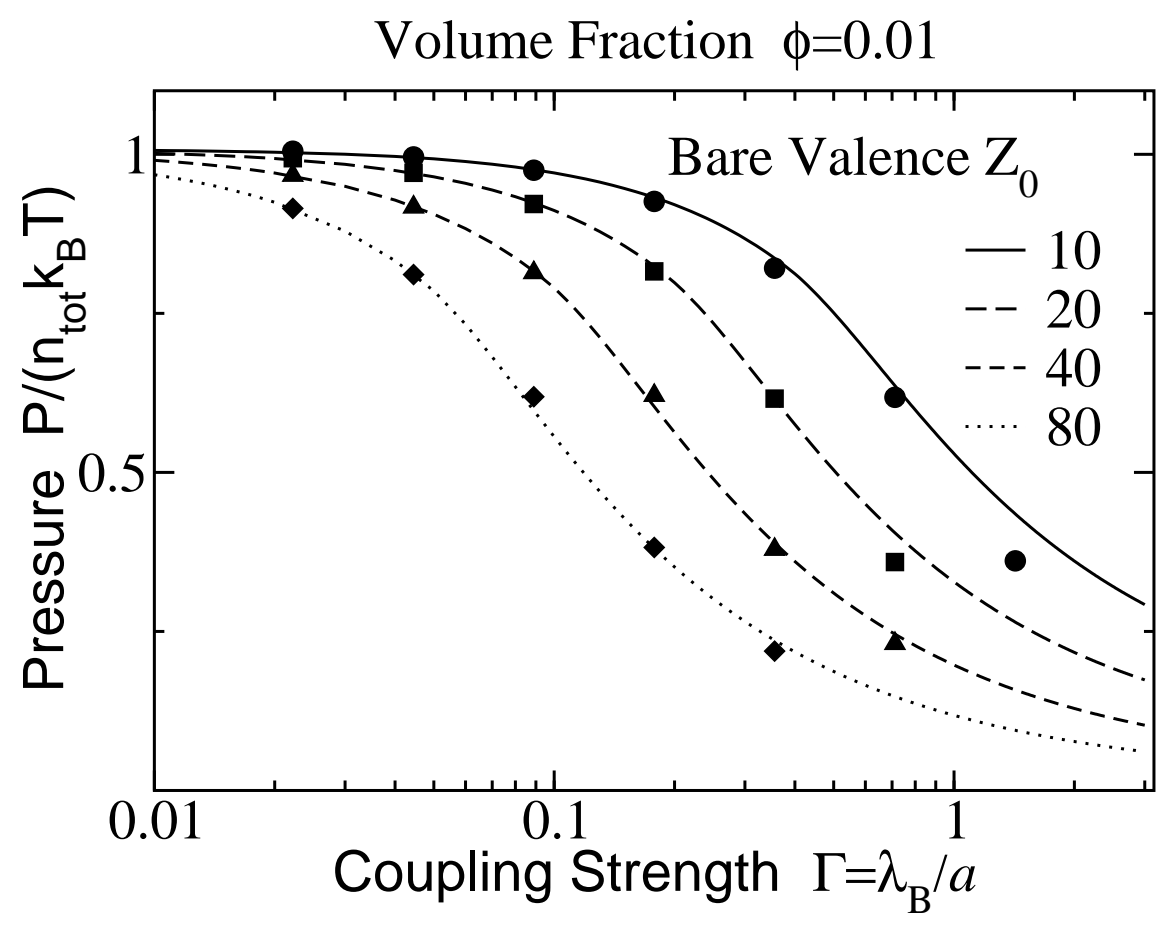

Figure 3. Pressure vs. electrostatic coupling constant $\Gamma \equiv \lambda_{B} / a$ for salt-free suspensions with colloid volume fraction $\phi=0.01$ and several bare valences $Z_{0}$. Predictions of the effective interaction model (curves) are compared with primitive model simulation data (symbols) [7].

that are strongly associated with the macroions, the renormalized theory includes most of the nonlinear response inherent in the PB equation. Comparably accurate results are obtained with a renormalized jellium theory [47, 48, 49]. The effective interaction model also predicts radial distribution functions in close agreement with simulations of the primitive model [41, 42].

\section{Outlook}

To illustrate the essence of coarse-grained modeling in a friendly context, the discussion in this chapter is limited to the primitive model of charged colloids, focusing on monodisperse suspensions of microspheres. For this system, the effective electrostatic interactions derived using the coarse-graining scheme described in Sec. 6] are relatively simple and lead to predictions for thermodynamic and structural properties that agree closely with the Poisson-Boltzmann cell model, detailed simulations of the primitive model, and experiment. Tremendous opportunities now lie ahead for extending the general methods outlined here and applying them to more complex systems, such as mixtures of colloids differing in size, shape, and charge, to suspensions of particles with anisotropic (patchy) charge distributions (e.g., 


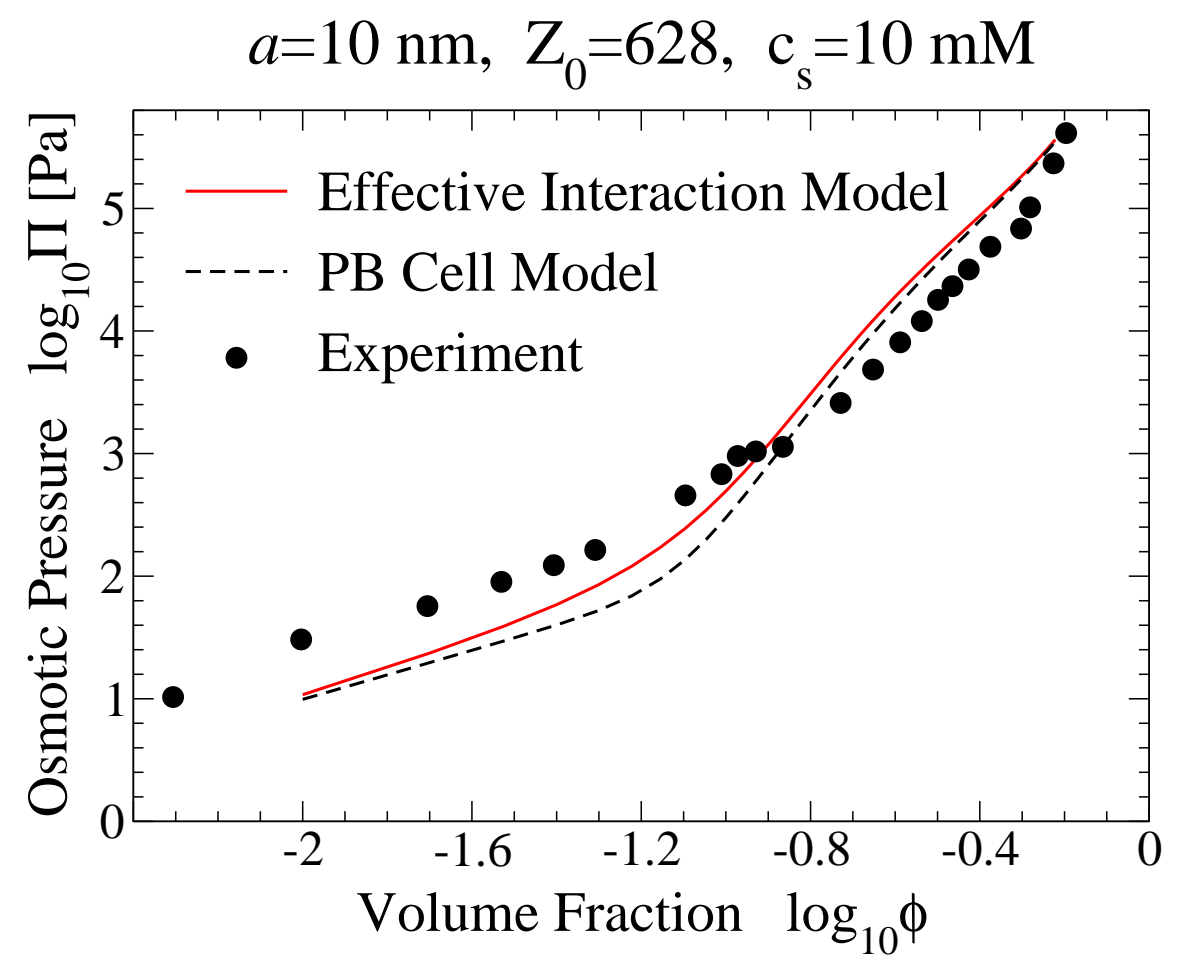

Figure 4. Osmotic pressure vs. colloid volume fraction for suspensions with macroion radius $a=10 \mathrm{~nm}$, bare valence $Z_{0}=628$, and salt concentration $c_{s}=10 \mathrm{mM}$. Predictions of the effective interaction model (solid curve) and the PB cell model (dashed curve) are compared with experimental data (symbols) [44, 45].

Janus particles), as well as to different interparticle interactions, such as dipolar interactions. As computing power grows, the demand for coarse-grained models will likely persist, to guide and interpret simulations and to facilitate exploration of increasingly complex materials.

\section{Acknowledgments}

It is a pleasure to thank Jun Kyung Chung and Sylvio May for helpful discussions. This work was supported by the National Science Foundation under Grant No. DMR-1106331.

[1] Evans, D. F. and Wennerström, H. (1999). The Colloidal Domain, $2^{\text {nd }}$ ed. (Wiley-VCH, New York).

[2] Israelachvili, J. (1992). Intermolecular and Surface Forces (Academic, London).

[3] Belloni, L. (2000). Colloidal interactions, J. Phys.: Condens. Matter 12, pp. R549-R587.

[4] Hansen, J. P. and Löwen, H. (2000). Effective interactions between electric double layers, Annu. Rev. Phys. Chem. 51, pp. 209-242.

[5] Likos, C. N. (2001). Effective interactions in soft condensed matter physics, Phys. Rep. 348, pp. 267-439.

[6] Denton, A. R. (2007). In Nanostructured Soft Matter: Experiment, Theory, Simulation and Perspectives, edited by A. V. Zvelindovsky (Springer, Dordrecht). 
[7] Linse, P. (2000). Structure, phase stability, and thermodynamics in charged colloidal solutions, J. Chem. Phys. 113, pp. 4359-4373.

[8] Brukhno, A. V., Åkesson, T., and Jönsson, B. (2009). Phase Behavior in Suspensions of Highly Charged Colloids, J. Phys. C: Solid State Phys. B 113, pp. 6766-6774.

[9] Hynninen, A.-P. and Panagiotopoulos, A. Z. (2007). Disappearance of the Gas-Liquid Phase Transition for Highly Charged Colloids, Phys. Rev. Lett. 98, pp. 1983011-4.

[10] Deserno, M. and Holm, C. (2001). In Electrostatic Effects in Soft Matter and Biophysics, vol. 46, NATO Advanced Studies Institute, Series II: Mathematics, Physics and Chemistry, edited by C. Holm et al. (Kluwer, Dordrecht).

[11] Denton, A. R. (2010). Poisson-Boltzmann theory of charged colloids: limits of the cell model for salty suspensions, J. Phys.: Condens. Matter 22, pp. 364108-1-12.

[12] Evans, R. (1992). In Inhomogeneous Fluids, edited by D. Henderson (Dekker).

[13] Evans, R. (1979). The nature of the liquid-vapour interface and other topics in the statistical mechanics of nonuniform, classical fluids, Adv. Phys. 28, pp. 143-200.

[14] Oxtoby, D. W. (1991). Crystallization of liquids: A density-functional approach, in Liquids, Freezing and Glass Transition, Les Houches session 51, edited by Hansen, J.-P., Levesque, D. and Zinn-Justin, J., 145-189 (North-Holland, Amsterdam).

[15] Löwen, H., Madden, P. A., and Hansen, J.-P. (1992). Description of Counterion Screening in Colloidal Suspensions, Phys. Rev. Lett. 68, pp. 1081-1084.

[16] Löwen, H., Madden, P. A., and Hansen, J.-P. (1993). Nonlinear counterion screening in colloidal suspensions, J. Chem. Phys. 98, pp. 3275-3289.

[17] Löwen, H. and Kramposthuber, G. (1993). Optimal Effective Pair Potential for Charged Colloids, Europhys. Lett. 23, pp. 673-678.

[18] Tehver, R., Ancilotto, F., Toigo, F., Koplik, J., and Banavar, J. R. (1999). Absence of many-body effects in interactions between charged colloidal particles, Phys. Rev. E 59, pp. R1335-R1338.

[19] Dobnikar, J., Chen, Y., Rzehak, R., and von Grünberg, H. H. (2003). Many-body interactions and the melting of colloidal crystals, J. Chem. Phys. 119, pp. 4971-4985.

[20] Dobnikar, J., Haložan, D., Brumen, M., von Grünberg, H. H., and Rzehak, R. (2004). PoissonBoltzmann Brownian dynamics of charged colloids in suspension, Comp. Phys. Comm. 159, pp. 73-92.

[21] Marcus, R. A. (1955). Calculation of Thermodynamic Properties of Polyelectrolytes, J. Chem. Phys. 23, pp. 1057-1068.

[22] Wennerström, H., Jönsson, B., and Linse, P. (1982). The cell model for polyelectrolyte systems. Exact statistical mechanical relations, Monte Carlo simulations, and the Poisson-Boltzmann approximation, J. Chem. Phys. 76, pp. 4665-4670.

[23] Deserno, M. and von Grünberg, H. H. (2002). Osmotic pressure of charged colloidal suspensions: A unified approach to linearized Poisson-Boltzmann theory, Phys. Rev. E 66, pp. 011401-1-15.

[24] van Roij, R. and Hansen, J.-P. (1997). Van der Waals-Like Instability in Suspensions of Mutually Repelling Charged Colloids, Phys. Rev. Lett. 79, pp. 3082-3085.

[25] Graf, H. and Löwen, H. (1998). Density jumps across phase transitions in soft-matter systems, Phys. Rev. E 57, pp. 5744-5753.

[26] van Roij, R., Dijkstra, M., and Hansen, J.-P. (1999). Phase diagram of charge-stabilized colloidal suspensions: van der Waals instability without attractive forces, Phys. Rev. E 59, pp. 2010-2025.

[27] Zoetekouw, B. and van Roij, R. (2006). Nonlinear Screening and Gas-Liquid Separation in Suspensions of Charged Colloids, Phys. Rev. Lett. 97, 258302-258305.

[28] Warren, P. B. (2000). A theory of void formation in charge-stabilised colloidal suspensions at low ionic strength, J. Chem. Phys. 112, 4683-4698.

[29] Grimson, M. J. and Silbert, M. (1991). A self-consistent theory of the effective interactions in chargestabilized colloidal dispersions, Mol. Phys. 74, 397-404. 
[30] Denton, A. R. (1999). Effective interactions and volume energies in charge-stabilized colloidal suspensions, J. Phys.: Condens. Matter 11, pp. 10061-10071.

[31] Denton, A. R. (2000). Effective interactions and volume energies in charged colloids: Linear response theory, Phys. Rev. E 62, pp. 3855-3864.

[32] Denton, A. R. (2007). Electroneutrality and phase behavior of colloidal suspensions, Phys. Rev. E 76, 051401-1-11.

[33] Hansen, J.-P. and McDonald, I. R. (1986). Theory of Simple Liquids, $2^{\text {nd }}$ edition (Academic, London).

[34] Derjaguin, B. V. and Landau, L. (1941). Acta Physicochimica (URSS) 14, 633.

[35] Verwey, E. J. W. and Overbeek, J. T. G. (1948). Theory of the Stability of Lyophobic Colloids (Elsevier, Amsterdam).

[36] Louis, A. A. (2002). Beware of density dependent pair potentials, J. Phys.: Condens. Matter 149187.

[37] Dobnikar, J., Castañeda-Priego, R., von Grünberg, H. H., and Trizac, E. (2006). Testing the relevance of effective interaction potentials between highly-charged colloids in suspension, New J. Phys. 8, 277.

[38] Castañeda-Priego, R., Rojas-Ochoa, L. F., Lobaskin, V., and Mixteco-Sánchez, J. C. (2006). Macroion correlation effects in electrostatic screening and thermodynamics of highly charged colloids, Phys. Rev. E 74, 051408-1-6.

[39] Trizac, E., Belloni, L., Dobnikar, J., von Grünberg, H. H., and Castañeda-Priego, R. (2007). Macroion virial contribution to the osmotic pressure in charge-stabilized colloidal suspensions, Phys. Rev. E $\mathbf{7 5}$, 011401-1-8.

[40] Rojas-Ochoa, L. F., Castañeda-Priego, R., Lobaskin, V., Stradner, A., Scheffold, F., and Schurtenberger, P. (2008). Density Dependent Interactions and Structure of Charged Colloidal Dispersions in the Weak Screening Regime, Phys. Rev. Lett. 100, 178304-1-4.

[41] Castañeda-Priego, R., Lobaskin, V., Mixteco-Sánchez, J. C., Rojas-Ochoa, L. F., and Linse, P. (2012). On the calculation of the structure of charge-stabilized colloidal dispersions using density-dependent potentials, J. Phys.: Condens. Matter 24, pp. 065102-1-7.

[42] Lu, B. and Denton, A. R. (2010). Charge Renormalization, Thermodynamics, and Structure, of Deionized Colloidal Suspensions, Commun. Comput. Phys. 7, pp. 235-249.

[43] Denton, A. R. (2008). Charge renormalization, effective interactions, and thermodynamics of deionized colloidal suspensions, J. Phys.: Condens. Matter 20, pp. 494230-1-8.

[44] Jönsson, B., Persello, J., Li, J., and Cabane, B. (2011). Equation of State of Colloidal Dispersions, Langmuir 27, 6606-6614.

[45] Chang, J., Lesieur, P., Delsanti, M., Belloni, L., Bonnet-Gonnet, C., Cabane, B. (1995). Structural and Thermodynamic Properties of Charged Silica Dispersions, J. Phys. C: Solid State Phys. 99, pp. 15993-16001.

[46] Alexander, S., Chaikin, P. M., Grant, P., Morales, G. J., and Pincus, P. (1984). Charge renormalization, osmotic pressure, and bulk modulus of colloidal crystals, J. Chem. Phys. 80, 5776-5781.

[47] Pianegonda, S., Trizac, E., and Levin, Y. (2007). The renormalized jellium model for spherical and cylindrical colloids, J. Chem. Phys. 126, pp. 014702-1-10.

[48] Colla, T. E., Levin, Y., and Trizac, E. (2009). A self-consistent renormalized jellium approach for calculating structural and thermodynamic properties of charge stabilized colloidal suspensions, J. Chem. Phys. 131, pp. 074115-1-6.

[49] Falcón-González, J. M. and Castañeda-Priego, R., (2010). Note: Renormalized jellium model for charged colloids revisited, J. Chem. Phys. 133, pp. 216101-1-3. 